\title{
Impact of left ventricular outflow tract ellipticity on the grading of aortic stenosis in patients with normal ejection fraction
}

Frédéric Maes ${ }^{1,2}$, Sophie Pierard ${ }^{1,2}$, Christophe de Meester ${ }^{1,2}$, Jamila Boulif ${ }^{1,2}$, Mihaela Amzulescu ${ }^{1,2}$, David Vancraeynest ${ }^{1,2}$, Anne-Catherine Pouleur ${ }^{1,2}$, Agnès Pasquet ${ }^{1,2}$, Bernhard Gerber $^{1,2}$ and Jean-Louis Vanoverschelde ${ }^{1,2^{*}}$ (D)

\begin{abstract}
Background: The pathophysiology of paradoxical low-gradient (LG) severe aortic stenosis (SAS) remains controversial. As low transvalvular flow has been implicated, we sought to investigate the impact of left ventricular outflow tract (LVOT) ellipticity on the estimation of the LV stroke volume, the calculation of the aortic valve area (AVA) by use of the continuity equation and on AS severity grading.

Methods: We studied 190 consecutive patients (mean age: $72 \pm 13$ years; male: 57\%) with SAS (indexed AVA $<0.6 \mathrm{~cm}^{2} / \mathrm{m}^{2}$ ) and preserved LV ejection fraction, including 120 patients with severe high gradient (HG) AS and 70 with severe paradoxical LG-AS. AS severity, LV volumes and LVOT ellipticity were assessed by 2D-Doppler echocardiography and cardiac magnetic resonance (CMR).

Results: The LVOT exhibited an elliptical shape on CMR images, with a shorter anterior-posterior than median-lateral diameter $(2.2 \pm 0.2$ vs $2.8 \pm 0.3 \mathrm{~cm}, p<0.01$ ). Accordingly, the LVOT area measured by planimetry was larger than by 2D-echocardiography, assuming a circular orifice $\left(4.9 \pm 0.9 \mathrm{~cm}^{2}\right.$ vs $\left.3.7 \pm 0.8 \mathrm{~cm}^{2}, p<0.01\right)$. Inputting the elliptical LVOT area into the continuity equation resulted in a $29 \%$ increase in the indexed AVA (from $0.41 \pm 0.09 \mathrm{~cm}^{2}$ to $0.54 \pm 0.10 \mathrm{~cm}^{2}$ ). Accordingly, 30 (43\%) patients with severe paradoxical LG-SAS were reclassified as having moderate AS. Similar results were obtained when considering 3D-echo for direct planimetry of the LVOT in a subset of 75 patients.
\end{abstract}

Conclusions: Our results confirm that the LVOT is elliptical in shape and that taking this parameter into account in the calculation of the AVA results in reclassification of $43 \%$ of patients with severe paradoxical LG-AS into moderate AS.

Keywords: Aortic stenosis, Gradient, Left ventricular outflow tract

\section{Background}

Several recent retrospective studies have indicated that in elderly patients, and particularly in elderly women, severe aortic stenosis (AS) is frequently associated with lower than expected mean transvalvular gradients, even in the presence of preserved left ventricular (LV) ejection fraction (EF) [1-3]. The term severe "paradoxical low-gradient (LG)" AS was recently coined to describe

\footnotetext{
* Correspondence: jean-louis.vanoverschelde@uclouvain.be

'Pôle de Recherche Cardiovasculaire, Institut de Recherche Expérimentale et

Clinique, Université Catholique de Louvain, Brussels, Belgium

${ }^{2}$ Division of Cardiology, Cliniques Universitaires Saint-Luc, Brussels, Belgium
}

this new form of severe AS [4] and to differentiate it from the well-recognized "low flow - low gradient" form seen in patients with LV dysfunction.

There is considerable debate as to the mechanisms underlying severe paradoxical LG-AS [1, 4-11]. Because its prognosis has been shown to be similar to that of moderate aortic stenosis $[1,10,11]$ and because it almost systematically progresses toward severe HG-AS overtime, $[10,12-14]$ we have recently postulated that paradoxical LG-AS might represent a transition stage between truly moderate AS (with low mean gradients and an indexed aortic valve area (AVA) $\geq 0.6 \mathrm{~cm}^{2} / \mathrm{m}^{2}$ ) and truly severe AS (with $\mathrm{HG}$ and AVAi $<0.6 \mathrm{~cm}^{2} / \mathrm{m}^{2}$ ). We further 
hypothesized that use of the continuity equation to calculate the AVA could explain why some patients transiently pass through a stage of severe paradoxical LG-AS during their disease progression [10]. There are indeed several reasons to believe that the use of the continuity equation leads to underestimation of the AVA. First, it measures the size of the functional orifice instead of that of the anatomical orifice, like direct planimetry or the Gorlin formula. This is because, in its simplified form, it neglects the coefficient of orifice contraction, a factor that compensates for the continuous convergence of fluid streamlines beyond a narrowed orifice. Under physiological flow conditions, the degree of underestimation of the anatomical orifice by the continuity equation is expected to be around 10-15\% [15]. Second, in daily clinical practice, the LV stroke volume, which is at the numerator in the continuity equation, is typically calculated by multiplying the velocity time integral of the LV outflow tract (LVOT) flow velocity by the cross-sectional area of the LVOT, the latter being calculated by measuring the anterior-posterior dimensions of the LVOT and assuming a circular orifice. Several studies have recently shown that the LVOT is rarely circular and most often exhibits an elliptical shape [16-20]. Depending on the degree of ellipticity, this is likely to result in significant underestimation of both LV stroke volume and calculated AVAs and hence in inconsistent grading of the true severity of AS.

The present study was designed to test the impact of LVOT ellipticity on the Doppler-echocardiographic estimation of LV stroke volume, AVA and AS severity grading, using cardiac magnetic resonance (CMR) as a reference standard. LVOT ellipticity was measured by direct planimetry of the aortic annulus using cardiovascular magnetic resonance (CMR). LVOT ellipcity was also measured by using transthoracic 3D-echocardiography in the subset of patient in whom 3D-volumetric datasets centered on the LVOT were available.

\section{Methods}

\section{Patient population}

Since January 2000, all patients with valvular heart disease referred to our Institution are enrolled into the prospective SALVARE (SAint-Luc VAlve Registry) registry. Baseline demographics, clinical features, as well as echocardiographic and CMR data are collected and stored in an electronic database. Subsequent clinical, echocardiographic and CMR data are regularly updated. From the registry database, we retrospectively selected all patients with native severe aortic stenosis who were included between May1 ${ }^{\text {st }}, 2005$ and February 30 ${ }^{\text {th }}, 2015$. To be selected, patients needed to display a preserved LVEF ( $\geq 50 \%$ ) and to have undergone CMR as part of their initial clinical workup. Patients with more than mild aortic regurgitation or more than trivial mitral regurgitation were not considered for inclusion.

\section{Transthoracic echocardiography (TTE)}

Echocardiographic data were obtained by use of commercially available ultrasound systems. All patients underwent a comprehensive examination, including 3-dimensional echocardiography when available. All tests were conducted by experienced sonographers. Images were analysed offline using the XCelera software (Philips Medical Systems, Andover, MA).

For assessment of AS, multiple transducer positions were systematically used to record peak aortic jet velocities [21]. The LVOT diameter was obtained from the parasternal long-axis view in mid-systole, parallel to the valve plane and immediately adjacent to the aortic leaflet insertion into the annulus [22]. The LVOT velocity was recorded from the apical window by placing the pulsedwave-Doppler sample volume in the outflow tract, proximal to the aortic valve. Proper positioning of the sample volume was ensured by verifying the presence of smooth spectral velocity curves associated with an aortic valve closing click. Care was taken to optimize the ultrasound beam - blood flow alignment and to avoid sampling in the transvalvular jet or the proximal flow convergence region by excluding velocity curves with spectral broadening at peak ejection. The maximal velocity across the aortic valve was measured with continuous-wave Doppler from multiple positions (apical, right parasternal, suprasternal and subxyphoidal). The highest velocity signal was used to calculate peak and mean gradients. The AVA was calculated by use of the continuity equation, assuming that the LVOT area had a circular shape. In case of atrial fibrillation, five consecutive beats were systematically averaged.

Severe AS was defined as an indexed AVA $<0.6 \mathrm{~cm}^{2} / \mathrm{m}^{2}$ and was further stratified into subgroups with high and paradoxically low transvalvular gradients, respectively in the presence of a mean transvalvular gradient $\geq$ and $<40 \mathrm{mmHg}$. Patients with severe paradoxical LG-AS were further stratified into subgroups with low flow and normal flow, respectively in the presence of an indexed stroke volume $\leq 35$ or $>35 \mathrm{~mL} / \mathrm{m}^{2}$ [4].

Three-dimensional echocardiographic datasets centered on the LVOT were available in a subset of 75 patients and analyzed using a QLab workstation (Philips Medical Systems, Andover, MA). After proper reorientation of the imaging plane, the true short axis of the LVOT was identified just below the aortic annulus. The LVOT area was then measured in midsystole by direct planimetry of the LVOT. Minimal and maximal diameters were also measured at the same level, to calculate the ellipticity index. 


\section{Cardiac magnetic resonance (CMR)}

CMR imaging was performed on a $1.5 \mathrm{~T}$ or $3 \mathrm{~T}$ magnet (Intera CV, Philips Medical Systems, Best, the Netherlands) using a five-element cardiac synergy coil for signal reception [22]. After localization of the heart using three-plane and oblique survey images, a three-chamber view and an oblique coronal view cine image of the LVOT were prescribed. These images were used as localizers to prescribe six contiguous cross-sectional cine images of the aortic valve between the LVOT and the tips of the aortic valve. The cine images were acquired using a multislice cine steady-state free precession (SSFP) pulse sequence during repeated breathholds. Slice thickness was $5 \mathrm{~mm}$ and slice spacing was $0 \mathrm{~mm}$. Data were stored in an electronic database and analyzed off-line using commercially available software (Osirix Viewer v.5.7, Pixmeo, Bernex, Switzerland). The LVOT contours were traced manually after zooming on the area of interest (Fig. 1). LVOT minimal and maximal diameters were measured at the same level, to calculate the ellipticity index. LV volumes and ejection fraction were computed semiautomatically with manual corrections. The LV stroke volume was calculated as the difference between LV end-diastolic and end-systolic volumes. Finally, the AVA was measured by direct planimetry of the maximal opening of the aortic valve tips in systole, as previously described [23].

\section{Fusion of Doppler with CMR data}

By combining hemodynamic echocardiographic data and LVOT area measured on CMR images, the fused indexed AVA was calculated introducing the CMR-derived LVOT area in the continuity equation.

\section{Statistical analysis}

All analyses were performed using the SPSS v19.0 (SPSS Inc., IBM, Chicago, IL). Continuous variables were expressed as mean \pm 1 SD when normally distributed and as median and range when non-normally distributed. Normality was assessed by use of the the Kolmogorov Smirnov test. Continuous variables were compared among groups using ANOVA when normally distributed or else using the Kruskall-Wallis test. Individual differences among groups were compared post-hoc using TukeyKramers test for normally distributed data with equal variances, the Games-Howell test for normally distributed data with unequal variances and the Mann-Whitney $U$ tests (with Bonferroni correction for multiple comparisons) for non-normally distributed data. Categorical variables

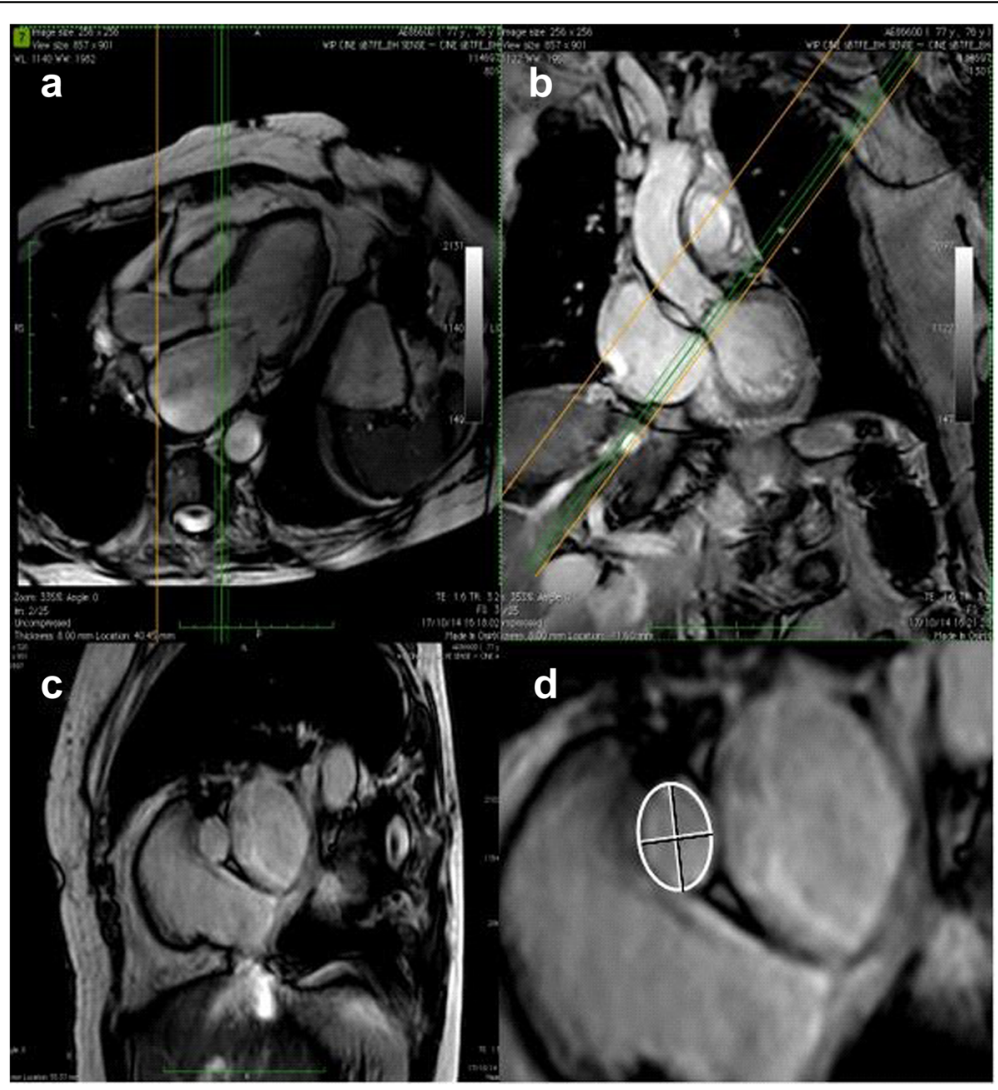

Fig. 1 Reprentatives examples of 2 orthogonal long-axis (panels $\mathbf{a}$ and $\mathbf{b}$ ) and the resulting short-axis (panels $\mathbf{c}$ and $\mathbf{d}$ ) images of the LVOT by CMR illustrating its elliptical shape 
were expressed as counts and percentages. Categorical variables of patients in different groups were compared using $\chi^{2}$ or the Fisher exact test. Correlation between CMR and echo data was evaluated by linear regression. Agreement between the two methods was assessed by Bland-Altman analysis. Intra- and interobserver variability for 2Dechocardiography, 3D-echocardiography and CMR data were assessed in a subgroup of 25 randomly selected patients by use of the intraclass correlation coefficient (ICC) and the Bland Altman method. All tests were two-sided and a $p$ value of $<0.05$ was considered indicative of a statistically significant difference.

\section{Results}

Among the 273 subjects who met the inclusion criteria, 83 patients were subsequently excluded because of incomplete or poor quality echocardiographic images $(n=43)$ or more than mild aortic regurgitation or trivial mitral regurgitation $(n=40)$. The final study population thus consisted of 190 patients; of which 120 displayed a mean transaortic pressure gradient $>40 \mathrm{mmHg}$ (HG-SAS) and 70 had a mean transaortic pressure gradient $\leq 40 \mathrm{mmHg}$ (paradoxical LG-SAS). Patients with severe paradoxical LG-AS were further stratified into groups with normal (NF, $n=45$ ) and low (LF, $n=25)$ transvalvular flow, using the previously described cut-off value of $35 \mathrm{~mL} / \mathrm{m}^{2}$ [4].

\section{Baseline clinical, hemodynamic and echocardiographic characteristics}

Are summarized in Tables 1 and 2. The three groups were comparable for most clinical and hemodynamic variables, with the exception of atrial fibrillation which was more prevalent in patients with severe paradoxical LF-LG-AS. From an echocardiographic point of view, patients with severe paradoxical LF-LG-AS exhibited smaller LV end-diastolic and end-systolic volume indices, lower indexed stroke volumes and smaller LVOT areas than patients from the two other subgroups. On the other hand, patients with severe paradoxical NF-LG-AS displayed larger indexed AVAs than patients from the two other subgroups. Similar results were obtained after exclusion of patients with atrial fibrillation.

\section{LVOT dimensions by 2D-echocardiography and CMR}

As shown in Fig. 1, the LVOT exhibited an elliptical shape on short-axis CMR images, with a shorter anteriorposterior than median-lateral diameter $(2.2 \pm 0.2$ vs $2.8 \pm$ $0.3 \mathrm{~cm}, p<0.001)$, yielding an averaged ellipticity index of $1.28 \pm 0.08$. Although the anterior-posterior LVOT dimensions by $2 \mathrm{D}$-echocardiography and CMR were quite similar $(2.2 \pm 0.2$ vs $2.2 \pm 0.2 \mathrm{~cm}, p=0.45)$, with a mean bias of $0.1 \pm 1.2 \mathrm{~mm}$ (Fig. 2a), the LVOT area measured by 2Dechocardiography, assuming a circular orifice, was significantly smaller than that measured by planimetry on CMR images $\left(3.7 \pm 0.8\right.$ vs $\left.4.9 \pm 0.9 \mathrm{~cm}^{2}, p<0.01\right)$ with a mean bias of $1.1 \pm 0.6 \mathrm{~cm}^{2}$ (Fig. 2b).

\section{Stroke volume and continuity equation-derived AVA using 2D-echocardiography and CMR LVOT areas}

As shown in Fig. 3, the indexed stroke volume measured with 2D-Doppler echocardiography, assuming a circular LVOT, was significantly lower than that measured by CMR $\left(42 \pm 9\right.$ vs $\left.51 \pm 11 \mathrm{mLs} / \mathrm{m}^{2}, p<0.01\right)$, with a mean bias of $10 \pm 11 \mathrm{mLs} / \mathrm{m}^{2}$. Similarly, the indexed AVA calculated by use of $2 \mathrm{D}$-Doppler echocardiography and the continuity equation was also smaller than the indexed AVA measured using CMR and direct planimetry of the anatomical orifice $\left(0.41 \pm 0.09\right.$ vs $0.54 \pm 0.10 \mathrm{~cm}^{2} / \mathrm{m}^{2}, p<$ 0.01 ), with a mean bias of $0.13 \pm 0.09 \mathrm{~cm}^{2} / \mathrm{m}^{2}$.

Inputting the CMR planimetered LVOT area into the calculation of the LV stroke volume resulted in a $29 \%$ increased of the indexed stroke volume (from $42 \pm 9$ to $54 \pm 11 \mathrm{~mL} / \mathrm{m} /{ }^{2}$ ) and indexed AVA (from $0.41 \pm 0.09 \mathrm{~cm}^{2}$ to $0.53 \pm 0.11 \mathrm{~cm}^{2} / \mathrm{m}^{2}$, all $p<0.01$ ). As a consequence, the

Table 1 Baseline clinical and hemodynamic characteristics in the different subgroups

\begin{tabular}{|c|c|c|c|c|}
\hline & $\begin{array}{l}\text { Severe } \\
\text { HG-SAS } \\
(n=120)\end{array}$ & $\begin{array}{l}\text { Severe } \\
\text { Paradoxical } \\
\text { NF-LG-AS } \\
(n=45)\end{array}$ & $\begin{array}{l}\text { Severe } \\
\text { Paradoxical } \\
\text { LF-LG-AS } \\
(n=25)\end{array}$ & $p$-value \\
\hline Age, yrs & $73 \pm 12$ & $74 \pm 14$ & $74 \pm 14$ & 0.44 \\
\hline Male gender, $n(\%)$ & 77 (64\%) & $21(47 \%)^{*}$ & $10(40 \%)^{*}$ & 0.02 \\
\hline Body surface area, $\mathrm{kg} / \mathrm{m}^{2}$ & $1.86 \pm 0,19$ & $1.81 \pm 0,21$ & $1.83 \pm 0,20$ & 0.83 \\
\hline Arterial hypertension, $n(\%)$ & $84(70 \%)$ & $38(84 \%)^{*}$ & $21(88 \%)^{*}$ & 0.046 \\
\hline Diabetes, $n(\%)$ & $20(17 \%)$ & $12(27 \%)$ & $6(24 \%)$ & 0.29 \\
\hline Hyperlipidemia, n (\%) & $86(72 \%)$ & $27(60 \%)$ & $24(96 \%)^{*}, \dagger$ & 0.01 \\
\hline Prior myocardial infarction, $n(\%)$ & $5(4 \%)$ & $7(16 \%)^{*}$ & $3(13 \%)$ & 0.04 \\
\hline Prior coronary revascularization, $n(\%)$ & $15(13 \%)$ & $5(11 \%)$ & $5(20 \%)$ & 0.49 \\
\hline Atrial fibrillation, $n(\%)$ & $2(2 \%)$ & $1(2 \%)$ & $5(20 \%)^{*}+t$ & $<0.01$ \\
\hline
\end{tabular}

${ }^{\circ} p<0.05$ vs HG-SAS; ${ }^{\dagger} p<0.05$ vs NF-PLG-SAS 
Table 2 Baseline hemodynamic, echocardiographic and CMR characteristics in the different subgroups

\begin{tabular}{|c|c|c|c|c|}
\hline & $\begin{array}{l}\text { Severe } \\
\text { HG-SAS } \\
(n=120)\end{array}$ & $\begin{array}{l}\text { Severe } \\
\text { Paradoxical } \\
\text { NF-LG-AS } \\
(n=45)\end{array}$ & $\begin{array}{l}\text { Severe } \\
\text { Paradoxical } \\
\text { LF-LG-AS } \\
(n=25)\end{array}$ & $p$-value \\
\hline \multicolumn{5}{|l|}{ Hemodynamic data } \\
\hline Heart rate, bpm & $68 \pm 10$ & $69 \pm 14$ & $76 \pm 16$ & 0.17 \\
\hline Systolic blood pressure, $\mathrm{mmHg}$ & $133 \pm 17$ & $141 \pm 22^{*}$ & $139 \pm 21$ & 0.70 \\
\hline Diastolic blood pressure, $\mathrm{mmHg}$ & $74 \pm 11$ & $76 \pm 11$ & $75 \pm 12$ & 0.88 \\
\hline \multicolumn{5}{|l|}{ CMR findings } \\
\hline Indexed LV EDV, $\mathrm{mL} / \mathrm{m}^{2}$ & $81 \pm 18$ & $73 \pm 18^{*}$ & $67 \pm 14^{*, \dagger}$ & $<0.01$ \\
\hline Indexed LV ESV, mL/m² & $28 \pm 10$ & $26 \pm 12$ & $25 \pm 8$ & 0.14 \\
\hline Indexed $\mathrm{SV}, \mathrm{mL} / \mathrm{m}^{2}$ & $54 \pm 10$ & $49 \pm 9$ & $43 \pm 10$ & $<0.01$ \\
\hline LV ejection fraction, \% & $59 \pm 5$ & $60 \pm 6$ & $57 \pm 5$ & 0.74 \\
\hline CMR LVOT diameter, mm & $22 \pm 2$ & $21 \pm 2$ & $21 \pm 2^{*}$ & 0.20 \\
\hline CMR LVOT area, $\mathrm{cm}^{2}$ & $5.0 \pm 1.0$ & $4.6 \pm 0.9^{*}$ & $4.6 \pm 0.7$ & 0.04 \\
\hline CMR LVOT ellipticity index & $1.28 \pm 0.08$ & $1.28 \pm 0.07$ & $1.30 \pm 0.10$ & 0.64 \\
\hline \multicolumn{5}{|l|}{ 2D-Doppler and Doppler findings } \\
\hline 2D-LVOT diameter, mm & $22 \pm 2$ & $22 \pm 2$ & $20 \pm 1^{*},+$ & 0.02 \\
\hline 2D-echo LVOT area, $\mathrm{cm}^{2}$ & $3.8 \pm 0.8$ & $3.7 \pm 0.6$ & $3.3 \pm 0.5^{*}$ & 0.02 \\
\hline 3D-echo LVOT area, $\mathrm{cm}^{2}$ & $5.3 \pm 1.0$ & $4.9 \pm 0.9$ & $4.6 \pm 0.9$ & 0.36 \\
\hline LVOT VTI, cm/s & $22 \pm 4$ & $22 \pm 3$ & $16 \pm 3^{*, t}$ & $<0.01$ \\
\hline 2D-indexed SV, $\mathrm{mL} / \mathrm{m}^{2}$ & $44 \pm 9$ & $42 \pm 5$ & $29 \pm 4^{*, t}$ & $<0.01$ \\
\hline Peak transaortic flow velocity, $\mathrm{cm} / \mathrm{s}$ & $472 \pm 49$ & $366 \pm 36^{*}$ & $344 \pm 42^{*, \dagger}$ & $<0.01$ \\
\hline Mean pressure gradient, mm Hg & $56 \pm 12$ & $32 \pm 6^{*}$ & $29 \pm 7^{*}, \dagger$ & $<0.01$ \\
\hline 2D-indexed AVA, $\mathrm{cm}^{2} / \mathrm{m}^{2}$ & $0.38 \pm 0.08$ & $0.49 \pm 0.06^{*}$ & $0.39 \pm 0.08^{\dagger}$ & $<0.01$ \\
\hline \multicolumn{5}{|l|}{ Fused data } \\
\hline Fused indexed SV, $\mathrm{mL} / \mathrm{m}^{2}$ & $57 \pm 10$ & $54 \pm 8^{*}$ & $41 \pm 5^{*, \dagger}$ & $<0.01$ \\
\hline Fused indexed AVA, $\mathrm{cm}^{2} / \mathrm{m}^{2}$ & $0.49 \pm 0.09$ & $0.62 \pm 0.10^{*}$ & $0.54 \pm 0.11^{*,+}$ & $<0.01$ \\
\hline
\end{tabular}

Abbreviations: $A V A$ aortic valve area, $E R O$ effective orifice area, $E D V$ end-diastolic volume, ESV end-systolic volume, $L V$ left ventricular ${ }^{*} p<0.05$ vs HG-SAS; ${ }^{\dagger} p<0.05$ vs NF-PLG-SAS
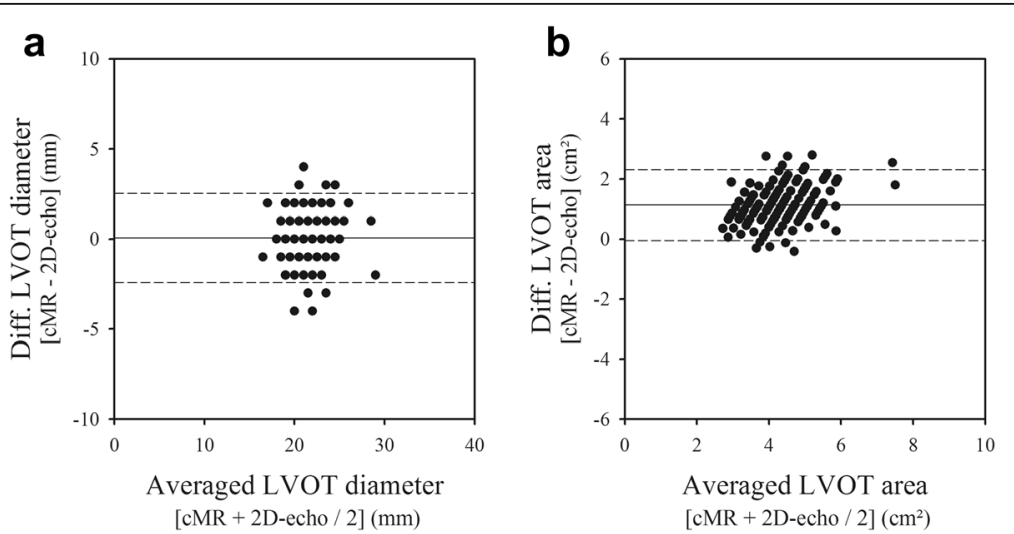

Fig. 2 Bland-Altman plots comparing 2D-echo and CMR measurements of the left ventricular outflow tract (LVOT) anterior-posterior diameters and cross-sectional area. On average, 2D-echo underestimated the LVOT area by 29\% compared with CMR 

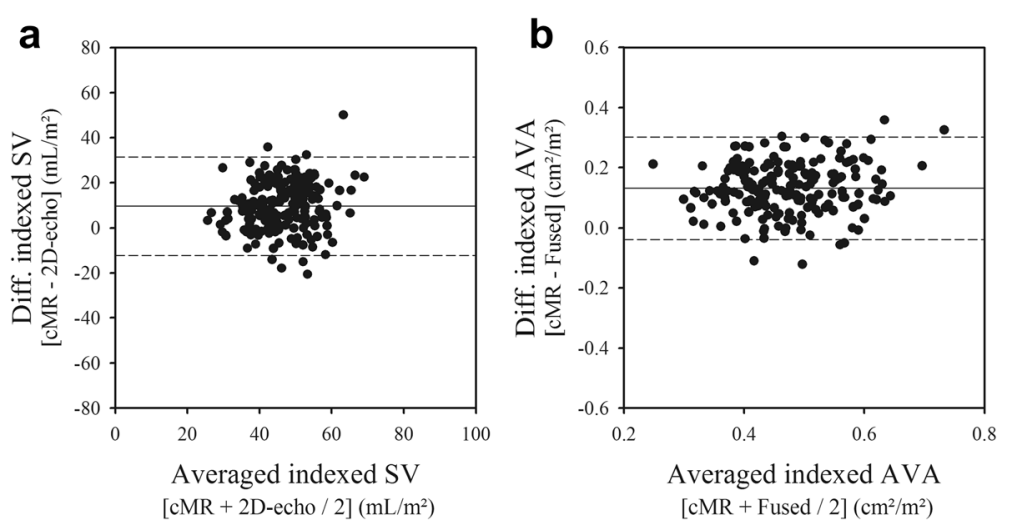

Fig. 3 Bland-Atman plots comparing 2D-echo and CMR measurements of indexed stroke volume (panel a) and of indexed AVA (panel b). On average, 2D-echo underestimated the indexed stroke volume and AVA by 29\% compared with CMR

systematic biases seen when assuming a circular LVOT almost completely disappeared (Fig. 4). This also resulted in a significant upward shift of the indexed AVA - mean gradient relationship (Fig. 5). Fitting this relationship to a non-linear function based on the formula $A V A_{i}=p / \sqrt{\Delta P}$ allowed calculation of the indexed AVA corresponding to a mean gradient of $40 \mathrm{mmHg}$ $\left(\mathrm{AVA}_{\mathrm{i} 40 \mathrm{mmHg}}\right)$. Using the CMR planimetered LVOT area increased the indexed $\mathrm{AVA}_{40 \mathrm{mmHg}}$ from $0.41 \pm 0.09$ to $0.59 \pm 0.11 \mathrm{~cm}^{2} / \mathrm{m}^{2}$.

\section{Impact of LVOT eccentricity on AS classification}

By using the fused indexed AVA, 49 patients with severe AS were reclassified as having moderate AS (indexed AVA $\geq 0.6 \mathrm{~cm}^{2} / \mathrm{m}^{2}$ ), including 19/120 HG-SAS (16\%), 5/25 paradoxical LF-LG-SAS (20\%) and 25/45 paradoxical NF-LG-SAS (55\%). Accordingly, the concordance between Doppler-echocardiography and CMR increased from 74 to $84 \%$, when defining severe AS as an indexed AVA $<0.6 \mathrm{~cm}^{2} / \mathrm{m}^{2}$, and from 84 to $93 \%$, when defining severe AS as either an indexed AVA $<0.6 \mathrm{~cm}^{2}$ / $\mathrm{m}^{2}$ or a MG $>40 \mathrm{mmHg}(p<0.01)$.

\section{D-echo vs CMR LVOT areas}

To test the ability of 3D-echocardiography to measure the true LVOT area and hence to compensate for the inherent limitations of 2D-echocardiography, we compared the planimetered LVOT areas by 3D-echo and CMR in a subset of 75 patients in who zoomed 3D-echo datasets centered on the LVOT were available. 3D-echo and CMR LVOT areas were similar $\left(5.1 \pm 1.0\right.$ vs $4.9 \pm 1.0 \mathrm{~cm}^{2}, p=$ 0.48 ) with a mean bias of $0.1 \pm 0.6 \mathrm{~cm}^{2}$. Inputting the 3D-echo or CMR planimetered LVOT areas into the continuity equation yielded similar indexed stroke volumes $\left(56 \pm 13\right.$ vs $\left.55 \pm 11 \mathrm{mLs} / \mathrm{m}^{2}, p=0.62\right)$ and indexed AVAs $\left(0.56 \pm 0.13\right.$ vs $\left.0.53 \pm 0.11 \mathrm{~cm}^{2}, p=0.22\right)$. Reclassification of AS severity based on 3D-echo and CMR derived planimetered LVOT areas yielded similar results.
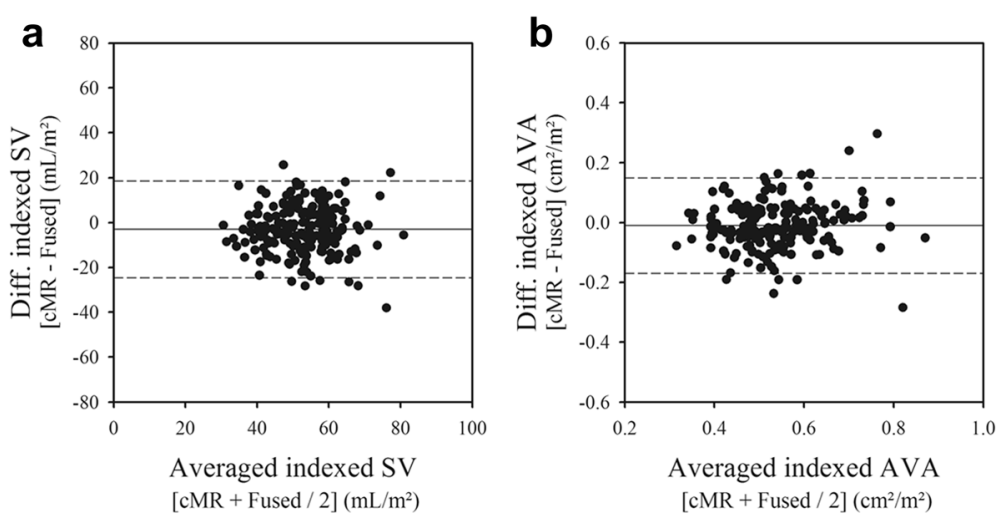

Fig. 4 Bland-Atman plots comparing fused and CMR measurements of indexed stroke volume (panel a) and of indexed AVA (panel b). On average, inputting the planimetered LVOT area into the calculation of the indexed stroke volume by 2D-echo, corrected the underestimation of the indexed stroke volume and AVA by 2D-echo as compared to CMR 

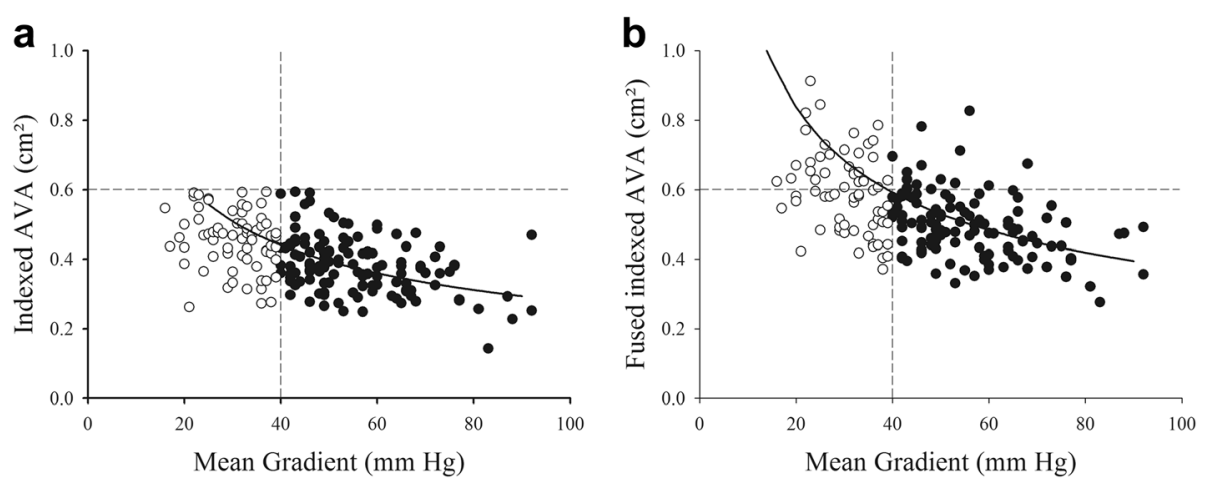

Fig. 5 Indexed AVA (panel a) and fused indexed AVA (panel b) vs. mean gradient among 120 with HG-SAS and 70 patients with paradoxical LG-SAS. The predicted values from the fitted curve of the study population are presented

\section{Variability of measurements}

LVOT planimetry measurements by CMR had an intraobserver variability (ICC) of 0.99 with a bias of $0.06 \pm 0.46 \mathrm{~cm}^{2}$ and interobserver variability of 0.95 with a bias of $-0.18 \pm 0.50 \mathrm{~cm}^{2}$. Intra and interobserver variability for $3 \mathrm{D}$-echo measurements were 0.86 and 0.94 with bias of $-0.04 \pm 0.048 \mathrm{~cm}^{2}$ and $0.03 \pm 0.98 \mathrm{~cm}^{2}$, respectively.

\section{Discussion}

The aim of the present study was to test the impact of LVOT ellipticity on the estimation of LV stroke volume, on the calculation of the AVA by use of continuity equation and on AS severity grading. CMR was used as the reference method for estimation of both LV stroke volume and AVA. Our results can be summarized as follows:

- In almost every patient, the LVOT exhibits an elliptical shape, with a larger transverse than anterior-posterior diameter;

- Inputting the CMR planimetered LVOT area into the continuity equation increased the indexed stroke volume and the indexed AVA by $29 \%$ and significantly reduced the bias between echocardiographic and CMR data;

- Based on the fused indexed AVAs, 55\% of patients with severe paradoxical NF-LG-AS, $20 \%$ of patients with severe paradoxical LF-LG-AS and $16 \%$ of patients with severe HG-AS were reclassified as having only moderate AS. Use of the fused indexed AVA improved the concordance between Doppler-echocardiography and CMR by an average of $10 \%$.

\section{Accuracy of Doppler echocardiography in measuring LV stroke volume}

Accurate measurement of the LV stroke volume is of paramount importance in the calculation of the AVA by use of the continuity equation [24]. Using 2D-Doppler echocardiography, this involves proper recording of the
LVOT diameter and subvalvular velocities. The LVOT diameter is usually measured in mid-systole on zoomed long-axis images. While it is usually easy to place the anterior measurement calliper onto the anterior aortic annulus, it is often more difficult to do so at the level of the posterior annulus, owing mainly to the lack of definite anatomical landmarks permitting its identification. Uncertainties regarding the exact location of the posterior aortic annulus likely explain the large variability reported in the literature for this measurement, which ranges between from 5 to $8 \%$ [24]. Since the LVOT diameter is squared for the calculation of its cross-sectional area, measurement variability is considered as one of the largest sources of error in stroke volume estimation. Yet, it is probably not the most important one. Several recent studies have indeed demonstrated that the LVOT is elliptical rather than circular in shape, leading to significant underestimation of the LVOT cross-sectional area [16-20].

The present study fully supports these previous findings. Our observations confirm that the LVOT is elliptically shaped and that its minor axis corresponds to that measured by 2D-echocardiography. Our data also indicate that the degree of underestimation of the LVOT area by 2D-echocardiography when assuming a circular orifice is as large as $29 \%$. Since the LVOT area is used to calculate the LV stroke volume, this parameter was proportionately underestimated. Other studies have previously reported similar underestimation of stroke volume by 2D-Doppler echocardiography [25-29].

\section{Impact of stroke volume underestimation on the mean gradient - aortic valve area relationship}

Because stroke volume is at the numerator in the continuity equation, calculated AVAs should be underestimated as well. Large degrees of underestimation of the AVA by the continuity equation in comparison of the planimetric AVA have been described before, [23] but were usually ascribed to the omission of the coefficient of orifice 
contraction in the simplified continuity equation. Our data indicate that the assumption of a circular LVOT is probably the main source of underestimation of both the LV stroke volume by 2D-Doppler echocardiography and the AVA by use of the continuity equation and that neglecting the coefficient of orifice contraction is probably less important.

As expected, underestimation of both the stroke volume and the AVA by conventional 2D-Doppler echocardiography had a major impact on the relationship between the AVA and the mean transaortic pressure difference. Gorlin and Gorlin were the first to systematically study this relationship [30]. Comparing hemodynamic data obtained by cardiac catheterization with surgical or autopsy findings, they elaborated a formula aimed at predicting the anatomical aortic valve area from hemodynamic measurements. Using this formula, and assuming a physiological mean transaortic flow rate of about $250 \mathrm{mLs} / \mathrm{sec}$, a mean transvalvular gradient of $40 \mathrm{mmHg}$ should correspond to an AVA of approximately $1 \mathrm{~cm}^{2}$. Minners et al. demonstrated that using 2D-Doppler echocardiography instead of catheterization to calculate the AVA results in a significant downward and leftward shift of the mean gradient valve area relationship, [2] so that a mean gradient of $40 \mathrm{mmHg}$ does not correspond anymore to an AVA of $1 \mathrm{~cm}^{2}$ but rather to an AVA of $0.81 \mathrm{~cm}^{2}$, when using the Gorlin formula, and $0.75 \mathrm{~cm}^{2}$, when using the continuity equation. This 19-25\% underestimation of the expected aortic valve area when using 2D-Doppler echocardiography explains why up to $40-45 \%$ of patients with a calculated AVA $<1 \mathrm{~cm}^{2}$ present with lower than expected mean transaortic pressure gradients, i.e. with a mean transaortic pressure difference $<40 \mathrm{mmHg}$ and why, at the opposite, very few patients with an aortic valve area $>1 \mathrm{~cm}^{2}$ present with a mean transaortic pressure difference $>40 \mathrm{mmHg}(<3 \%)$. The present study confirms and extends these observations. In line with previous studies, the proportion of patients presenting with lower than expected mean transaortic pressure gradient was as high as $37 \%$. Based on the mean gradient gradient - AVA relationship, a mean transvalvular gradient of $40 \mathrm{mmHg}$ was found to correspond to an indexed AVA of $0.44 \mathrm{~cm}^{2} / \mathrm{m}^{2}$ $\left(\mathrm{AVA}_{40 \mathrm{mmHg}}\right.$ ). Correcting for LVOT ellipticity reduced the proportion of patients presenting with lower than expected mean gradients to $25 \%$ and increased the indexed AVA $_{40 \mathrm{mmHg}}$ to $0.59 \mathrm{~cm}^{2} / \mathrm{m}^{2}$.

\section{Impact of LVOT ellipticity on AS classification}

Correcting the hemodynamic equations for the LVOT ellipticity also had a profound impact on the classification of AS patients into the different AS subgroups. Indeed, as many as $25 \%$ of patients with initially severe AS were reclassified as having moderate AS, including 16\% of patients with severe HG-AS, $20 \%$ of patients with severe paradoxical LF-LG-AS and 55\% of patients with severe paradoxical NF-LG-AS. As a consequence, the concordance between Doppler-echocardiography and CMR improved from 74 to $84 \%$. Similar results were recently reported by other investigators, using either multidetector computed tomography [31] or CMR [32].

Reclassification of patients with severe paradoxical NF-LG-AS into moderate AS may have important therapeutic implications since previous studies have shown that the survival of these patients was quite similar to that of patients with moderate AS. Their valves have also been shown to contain less calcium than those from patients with severe HG-AS, which further support the contention that they most probably do not exhibit truly severe AS [33, 34].

By contrast, only a minority of patients with severe paradoxical LF-LG-AS were reclassified as having moderate AS when correcting for LVOT ellipticity. Although this could indicate that they suffer from a more severe form of AS, as would be suggested by their worse outcome in some of the previously published series, $[4,8,9]$ one cannot exclude the possibility that this could simply be related to their low-flow state and the inability for their LV to open up an otherwise moderately stenotic aortic valve. This hypothesis would be consistent with their relatively low calcium content as noted by several previous investigators [31, 32].

\section{D-echocardiography to assess LVOT ellipticity}

Several studies have demonstrated the accuracy of 3Dechocardiography, and particularly 3D-transesophgeal echocardiography to delineate the elliptical shape of the LVOT and to measure its true cross-sectional area $[35,36]$. Our study is amongst the first to demonstrate that such measurements are feasible and accurate by use of 3D transthoracic echocardiography as well. Our data further demonstrate that 3D transthoracic echo measurements of the LVOT area compare favourably with those obtained by CMR and allow alleviating the underestimation of both the LV stroke volume and the AVA by conventional 2D-Doppler echocardiography. Accordingly, 3D-echo allowed a similar proportion of patients with severe AS to be reclassified as having moderate AS as compared with CMR.

\section{Study limitations}

Our study has several limitations that should be acknowledged. First, this is an observational study based on the a posteriori selection of patients having undergone CMR as part of their initial workup as well as on the retrospective analysis of the CMR data. Our results thus need to be confirmed in prospective studies. Second, planimetry and continuity equation-based AVAs are not identical. As discussed above, the first measures the anatomic valve area 
and the second measures the effective orifice area at the level of the vena contracta. In theory, they differ by a factor known as the coefficient of orifice contraction, which compensates for the continuous convergence of fluid streamlines beyond a stenotic orifice. Although fluid dynamics studies have indicated that neglecting this coefficient could lead to a $10-15 \%$ underestimation of the true anatomical orifice when using the continuity equation, our date indicate that this is certainly not the only factor to be considered and that underestimation of the LVOT area plays, at least, an equally important role. Finally, planimetry of the LVOT by 3D-echo was only available in 75 patients. The accuracy of this measurement should thus be confirmed in larger cohorts.

\section{Conclusions}

Our results confirm that the LVOT is elliptically shaped and that failure to take this characteristic into consideration when calculating the LV stroke volume and the AVA results in a $29 \%$ underestimation of these parameters as compared to CMR. Consequently, as many as $25 \%$ of patients with severe AS are reclassified as having only moderate AS when inputting the correct LVOT area into the continuity equation. This is particularly true in patients with severe paradoxical NF-LG-AS, of whom about $2 / 3$ are reclassified as having only moderate AS.

\section{Abbreviations}

3D: Three-dimensional; AS: Aortic stenosis; AVA: Aortic valve area; AVAi: Indexed aortic valve area; CMR: Cardiovascular magnetic resonance; HG: High gradient; LG: Low gradient; LV: Left ventricular; LVEF: Left ventricular ejection fraction; LVOT: Left ventricular outflow tract

\section{Acknowledgements}

NA.

\section{Funding}

B. Gerber is supported by the Fonds de Recherche Clinique (Cliniques universitaires Saint-Luc, Brussels, Belgium. A-C Pouleur is supported by the Fonds National de la Recherche Scientifique (FNRS, Brussels, Belgium).

\section{Availability of data and materials}

The datasets used and/or analysed during the current study available from the corresponding author on reasonable request.

\section{Authors' contributions}

All authors substantially contributed to conception and design and interpretation of data. All were involved in revising the manuscript critically and gave final approval of the version to be published. Each author should have participated sufficiently in the work to take public responsibility for appropriate portions of the content. FM acquired and analyzed the data. $\mathrm{CdM}$ made the statistical analyses. All authors read and approved the final manuscript.

\section{Authors' information}

NA.

\section{Competing interests}

The authors declare that they have no competing interests.

\section{Consent for publication}

NA.

\section{Ethics approval and consent to participate}

Inclusion of patients into the SALVARE registry was approved by the Commission d'éthique hospitalo-facultaire. Patients gave their written consent for being included in the SALVARE registry.

Received: 9 December 2016 Accepted: 16 February 2017

Published online: 15 March 2017

\section{References}

1. Jander N, Minners J, Holme I, Gerdts E, Boman K, Brudi P, Chambers JB, Egstrup K, Kesäniemi YA, Malbecq W, Nienaber CA, Ray S, Rossebø A, Pedersen TR, Skjærpe T, Willenheimer R, Wachtell K, Neumann FJ, Gohlke-Bärwolf C. Outcome of patients with low-gradient "severe" aortic stenosis and preserved ejection fraction. Circulation. 2011;123:887-95.

2. Minners J, Allgeier M, Gohlke-Baerwolf C, Kienzle R, Neumann F, Jander N. Inconsistencies of echocardiographic criteria for the grading of aortic valve stenosis. Eur Heart J. 2008;29:1043-8.

3. Minners J, Allgeier M, Gohlke-Baerwolf C, Kienzle RP, Neumann FJ, Jander N. Inconsistent grading of aortic valve stenosis by current guidelines: haemodynamic studies in patients with apparently normal left ventricular function. Heart. 2010;96:1463-8.

4. Hachicha Z, Dumesnil JG, Bogaty P, Pibarot P. Paradoxical low-flow, lowgradient severe aortic stenosis despite preserved ejection fraction is associated with higher afterload and reduced survival. Circulation. 2007:115:2856-64.

5. Dumesnil JG, Pibarot P, Carabello B. Paradoxical low flow and/or low gradient severe aortic stenosis despite preserved left ventricular ejection fraction: implications for diagnosis and treatment. Eur Heart J. 2010;31:281-9.

6. Mehrotra P, Jansen K, Flynn AW, Tan TC, Elmariah S, Picard MH, Hung J. Differential left ventricular remodelling and longitudinal function distinguishes low flow from normal-flow preserved ejection fraction lowgradient severe aortic stenosis. Eur Heart J. 2013;34:1906-14.

7. Adda J, Mielot C, Giorgi R, Cransac F, Zirphile X, Donal E, Sportouch-Dukhan C, Reant P, Laffitte S, Cade S, Le Dolley Y, Thuny F, Touboul N, Lavoute C, Avierinos JF, Lancellotti P, Habib G. Low-flow, low-gradient severe aortic stenosis despite normal ejection fraction is associated with severe left ventricular dysfunction as assessed by speckle-tracking echocardiography: a multicenter study. Circ Cardiovasc Imaging. 2012:5:27-35.

8. Clavel MA, Dumesnil JG, Capoulade R, Mathieu P, Sénéchal M, Pibarot P. Outcome of patients with aortic stenosis, small valve area, and low-flow, low-gradient despite preserved left ventricular ejection fraction. J Am Coll Cardiol. 2012;60:1259-67.

9. Lancellotti P, Magne J, Donal E, Davin L, O'Connor K, Rosca M, Szymanski C, Cosyns B, Piérard L. Clinical outcome in asymptomatic severe aortic stenosis: insights from the new proposed aortic stenosis grading classification. J Am Coll Cardiol. 2012:59:235-43.

10. Maes F, Boulif J, de Meester C, Melchior J, Gerber B, Vancraeynest D, Pouleur A-C, Lazam S, Pasquet A, Vanoverschelde J-L. Natural history of paradoxical low gradient « severe » aortic stenosis. Circ Cardiovasc Imaging. 2014;7:714-22.

11. Tribouilloy C, Rusinaru D, Maréchaux S, Castel AL, Debry N, Maizel J, Mentaverri R, Kamel S, Slama M, Lévy F. Low-gradient, low-flow severe aortic stenosis with preserved left ventricular ejection fraction: characteristics, outcome, and implications for surgery. J Am Coll Cardiol. 2015;65:55-66.

12. Rezzoug N, Vaes B, Pasquet A, Gerber BL, de Meester C, Van Pottelbergh G, Adriaensen W, Matheï C, Degryse J, Vanoverschelde J-L. Prevalence and prognosic impact of valve area - gradient patterns in patients $>80$ years of age with moderate to severe aortic stenosis. From the Prospective BELFRAll study. Am J Cardiol. 2015;116:925-32.

13. Tribouilloy C, Rusinaru D, Charles V, Boulif J, Maes F, Lévy F, Pasquet A, Maréchaux S, Vanoverschelde J-L. Progression of low-gradient, Low-flow, severe aortic stenosis with preserved left ventricular ejection fraction. Am J Cardiol. 2015;116:612-7.

14. Dahl JS, Eleid MF, Pislaru SV, Scott CG, Connolly HM, Pellikka PA. Development of paradoxical low-flow, low-gradient severe aortic stenosis. Heart. 2015;101:1015-23.

15. Yoganathan AP, Cape EG, Sung HW, Williams FP, Jimoh A. Review of hydrodynamic principles for the cardiologist: applications to the study of blood flow and jets by imaging techniques. J Am Coll Cardiol. 1988:12:1344-52.

16. Delgado V, Ng AC, van de Veire NR, van der Kley F, Schuijf JD, Tops LF, de Weger A, Tavilla G, de Roos A, Kroft LJ, Schalij MJ, Bax JJ. Transcatheter aortic valve implantation: role of multi-detector row computed tomography to 
evaluate prosthesis positioning and deployment in relation to valve function. Eur Heart J. 2010;31:1114-23.

17. Messika-Zeitoun D, Serfaty JM, Brochet E, Ducrocq G, Lepage L, Detaint $D$, Hyafil F, Himbert D, Pasi N, Laissy JP, lung B, Vahanian A. Multimodal assessment of the aortic annulus diameter: implications for transcatheter aortic valve implantation. J Am Coll Cardiol. 2010;55:186-94.

18. Ng AC, Delgado V, van der Kley F, Shanks M, van de Veire NR, Bertini M, Nucifora G, van Bommel RJ, Tops LF, de Weger A, Tavilla G, de Roos A, Kroft LJ, Leung DY, Schuijf J, Schalij MJ, Bax JJ. Comparison of aortic root dimensions and geometries before and after transcatheter aortic valve implantation by 2 - and 3-dimensional transesophageal echocardiography and multislice computed tomography. Circ Cardiovasc Imaging. 2010;3:94-102.

19. De Vecchi C, Caudron J, Dubourg B, Pirot N, Lefebvre V, Bauer F, Eltchaninoff $\mathrm{H}$, Dacher JN. Effect of the ellipsoid shape of the left ventricular outflow tract on the echocardiographic assessment of aortic valve area in aortic stenosis. J Cardiovasc Comput Tomogr. 2014;8:52-7.

20. Doddamani S, Grushko MJ, Makaryus AN, Jain VR, Bello R, Friedman MA, Ostfeld RJ, Malhotra D, Boxt LM, Haramati L, Spevack DM. Demonstration of left ventricular outflow tract eccentricity by 64-slice multi-detector $\mathrm{CT}$. Int J Cardiovasc Imaging. 2009;25:175-81.

21. Baumgartner H, Hung J, Bermejo J, Chambers JB, Evangelista A, Griffin BP, lung B, Otto CM, Pellikka PA, Quinones M. American Society of Echocardiography and European Association of Echocardiography. Echocardiographic assessment of valve stenosis: EAE/ASE recommendations for clinical practice. J Am Soc Echocardiogr. 2009;22:1-23.

22. Barone-Rochette G, Piérard S, Seldrum S, de Meester de Ravenstein C, Melchior J, Maes F, Pouleur AC, Vancraeynest D, Pasquet A, Vanoverschelde JL, Gerber BL. Aortic valve area, stroke volume, left ventricular hypertrophy, remodeling and fibrosis in aortic stenosis assessed by cardiac MRl: comparison between high and low gradient, and normal and low flow aortic stenosis. Circ Cardiovasc Imaging. 2013;6:1009-17.

23. Pouleur A-C, le Polain de Waroux J-B, Pasquet A, Vanoverschelde J-L, Gerber BJ. Aortic valve area assessment: multidetector CT compared with cine MR imaging and transthoracic and transesophageal echocardiography. Radiology. 2007;244:745-54.

24. Chambers JB, Vanoverschelde JL. Replacement heart valves. In: Galiuto L, editor. The EAE Textbook of Echocardiography. Oxford: Oxford University Press; 2011. p. 271-88.

25. Dubin J, Wallerson DC, Cody RJ, Devereux RB. Comparative accuracy of Doppler echocardiographic methods for clinical stroke volume determination. Am Heart J. 1990;120:116-23.

26. Loeppky JA, Hoekenga DE, Greene ER, Luft UC. Comparison of noninvasive pulsed Doppler and Fick measurements of stroke volume in cardiac patients. Am Heart J. 1984;107:339-46.

27. Fast JH, van den Merkhof L, Blans W, van Leeuwen K, Uijen G. Determination of cardiac output by single gated pulsed Doppler echocardiography. Int J Cardiol. 1988:21:33-42

28. Ihlen $H$, Myreng $Y$. Interobserver variability of the aortic orifice area measured by echocardiography. J Cardiovasc Technol. 1989:8:75-8.

29. Evangelista A, Garcia-Dorado D, Garcia Del Castillo H, Gonzalez-Alujas T, Soler-Soler J. Cardiac index quantification by Doppler ultrasound in patients without left ventricular outflow tract abnormalities. J Am Coll Cardiol. 1995:25:710-6.

30. Gorlin R, Gorlin SG. Hydraulic formula for calculation of the area of the stenotic mitral valve, other cardiac valves, and central circulatory shunts. Am Heart J. 1951;41:1-29.

31. Kamperidis $\mathrm{V}$, van Rosendael PJ, Katsanos $\mathrm{S}$, van der Kley F, Regeer M, Al Amri I, Sianos G, Marsan NA, Delgado V, Bax JJ. Low gradient severe aortic stenosis with preserved ejection fraction: reclassification of severity by fusion of Doppler and computed tomographic data. Eur Heart J. 2015;36:2087-96.

32. Chin CW, Khaw HJ, Luo E, Tan S, White AC, Newby DE, Dweck MR. Echocardiography underestimates stroke volume and aortic valve area: implications for patients with small-area low-gradient aortic stenosis. Can J Cardiol. 2014;30:1064-72

33. Clavel MA, Messika-Zeitoun D, Pibarot P, Aggarwal S, Malouf J, Araoz P, Michelena H, Cueff C, Larose E, Capoulade R, Vahanian A, Enriquez-Sarano M. The complex nature of discordant severe calcified aortic valve disease grading: new insights from combined Doppler-echocardiographic and computed tomographic study. J Am Coll Cardiol. 2013;62:2329-38.

34. Clavel MA, Ennezat PV, Maréchaux S, Dumesnil JG, Capoulade R, Hachicha Z, Mathieu P, Bellouin A, Bergeron S, Meimoun P, Arsenault M, Le Tourneau T,
Pasquet A, Couture C, Pibarot P. Stress echocardiography to assess stenosis severity and predict outcome in patients with paradoxical low-flow, low-gradient aortic stenosis and preserved LVEF. J Am Coll Cardiol Img. 2013;6:175-83.

35. Gaspar T, Adawi S, Sachner R, Asmer I, Ganaeem M, Rubinshtein R, Shiran A. Three-dimensional imaging of the left ventricular outflow tract: impact on aortic valve area estimation by the continuity equation. J Am Soc Echocardiogr. 2012;25:749-57.

36. Doddamani S, Bello R, Friedman MA, Banerjee A, Bowers Jr JH, Kim B, Vennalaganti PR, Ostfeld RJ, Gordon GM, Malhotra D, Spevack DM. Demonstration of left ventricular outflow tract eccentricity by real time 3D echocardiography: implications for the determination of aortic valve area. Echocardiography. 2007;24:860-6.

\section{Submit your next manuscript to BioMed Central and we will help you at every step:}

- We accept pre-submission inquiries

- Our selector tool helps you to find the most relevant journal

- We provide round the clock customer support

- Convenient online submission

- Thorough peer review

- Inclusion in PubMed and all major indexing services

- Maximum visibility for your research

Submit your manuscript at www.biomedcentral.com/submit
Biomed Central 